\title{
Alcohol markers in hair: an issue of interpretation.
}

The analysis of hair samples to show evidence of our long-term exposure to drugs and alcohol has come a long way since the early research in the 1990s. It is now fairly commonplace for scientists to analyse hair to reveal evidence of how much you drink, whether you take drugs, and even just how stressed you are. Technological advances in mass spectrometry mean that we can now routinely identify some compounds in hair at levels less than a picogram (per milligram of hair). Despite these remarkable advances, toxicologists still face a major issue when analysing samples to provide retrospective history of exposure to drugs and alcohol: what do the results of hair testing really mean, and is hair actually the best matrix to provide the evidence required? To address this, we need to examine whether the concept of an analytical 'cut-off' is still relevant for all compounds, and consider whether fingernails might prove to be a helpful alternative matrix in the future.

The use of hair analysis to provide evidence of alcohol consumption or abstinence is a good example of the issue. It has been demonstrated [1] that alcohol metabolites such as ethyl glucuronide (EtG) and fatty acid ethyl esters (FAEE) can be detected in our hair following alcohol consumption. Such tests are frequently used in criminal and civil cases worldwide, and there is sometimes dispute as to what the results of hair alcohol testing signify [2].

The interpretation of EtG or FAEE hair testing is complex, with issues of false positives, false negatives, and sometimes a difficulty in proposing whether or not alcohol has actually been consumed. The accepted methodology makes use of 'cut-off' values. This is a value which the hair testing result is compared against. Results below the cut-off are reported as 'not detected' or 'negative', whereas results falling above the cut-off would be considered as 'positive' or 'detected'. When attempting to detect the presence of illegal drugs in hair, for example, the use of a cut-off is simple and practical. The drug in question is illegal, so theoretically any detectable level of the drug in hair is of interest. A careful approach is required to consider issues of contamination, but in general a cut-off can safely be applied in such cases, at a level which lowers the risk of false positives and gives a level of confidence in the results.

When the target of a hair test is an alcohol marker though, interpreting the results is far more complex. Alcohol is legal in many countries, therefore often the question will need to address not just 'was alcohol consumed' but importantly 'how much alcohol was consumed'. This is a far more complex question. From hair analysis, these questions cannot be answered definitively in terms of a number of units, but instead a consensus has been reached [3] whereby a cut-off value has been established to suggest abstinence, and a higher cut-off used to suggest chronic excessive alcohol consumption. Current guidance [3] suggests that a cut-off for EtG of less than $7 \mathrm{pg} / \mathrm{mg}$ does not contradict self-reported abstinence of a person during the corresponding time period before sampling, and EtG concentrations higher than $30 \mathrm{pg} / \mathrm{mg}$ strongly suggest chronic excessive alcohol consumption. Whilst this is a practical solution for interpretation, it is not always appropriate, and certainly does not provide as much information as is necessary to make an informed judgement. For example, an EtG test result at $29 \mathrm{pg} / \mathrm{mg}$ has a different interpretative value than another test result at $31 \mathrm{pg} / \mathrm{mg}$, yet are only separated by a tiny margin.

Perhaps an alternative system to interpret this data is required. It would be more useful to evaluate the alcohol metabolite concentration and then indicate how likely it is that someone is a heavy drinker, or abstinent, given the concentration found in the hair, and not only relying on whether or not the concentration is above or below certain cut-offs. It is accepted that there is no direct correlation between hair alcohol metabolite concentration, and the level of alcohol consumed which allows a precise indication of alcohol consumption to be estimated. However, using the present cutoff based system, we are missing valuable data by not interpreting the significance of how far above 
or below a cut off a concentration may be. For instance, there may be a significant difference in the alcohol intake needed to produce EtG hair concentrations of 28,50 and $120 \mathrm{pg} / \mathrm{mg}$ respectively. We need to consider the value of this data, and through calculation we could propose just how much more likely it is that a person may drink heavily given an EtG concentration of $31 \mathrm{pg} / \mathrm{mg}$, than a concentration of $131 \mathrm{pg} / \mathrm{mg}$ for example.

This approach utilises Bayesian theory and relies on likelihood ratios. It is a common approach for the evaluation of forensic DNA evidence, and widely adopted in that field. The approach has been proposed [4-6] as an interpretation system for alcohol markers before, but uptake in the forensic community is minor. Using such a system, the strength of the evidence can be calculated, taking into account factors that contribute to false positives and false negatives. More research is needed to make the system a viable alternative to the cut-off system currently employed for alcohol marker hair analysis. Toxicology laboratories though need to be open to such change and embrace the value it could bring to the interpretation of their data.

One of the main barriers to a wider adoption of likelihood ratios for hair alcohol marker interpretation is the lack of data. The system works well in the field of forensic DNA profiling due to the prevalence of allele frequency data. A similar sort of data reserve is required for hair analysis. Perhaps if scientists pool resources, through the facilitating medium of the Society of Hair Testing, we can collectively produce a large data set of alcohol marker concentrations with accompanying self-reported alcohol consumption data. If available as an open access dataset, this would be an excellent starting point to move forward the research in this area.

Interpreting hair alcohol marker concentrations can be difficult, and the problem can be compounded when asked to provide evidence on the question of abstinence. It is often the case that individuals may drink alcohol moderately, yet test negative for EtG and FAEEs. The issue here is the sensitivity of hair to pick up such low traces of the alcohol markers. Research is underway in our laboratory to investigate whether fingernails might be a better sample to test in cases where we need to prove abstinence from alcohol. It has been shown [7] that fingernails may incorporate significantly more ethyl glucuronide (a metabolite of alcohol) than hair samples. Fingernails may therefore be more sensitive than hair, and could be able to better distinguish between low levels of drinking and complete abstinence. The issue here will again be one of interpretation. Whilst fingernails may incorporate a higher concentration of the alcohol markers, lending the matrix the advantage of sensitivity, the downside will be the lack of understanding of growth rate and the difficulty of associating alcohol consumption with a defined timeline. It is accepted that head hair grows at an average of $1 \mathrm{~cm}$ per month, giving sections of hair the ability to correlate alcohol marker concentrations with defined periods of time. We do not have the data yet to be able to apply this with fingernail samples. As always, more research is needed in this area.

\section{Compliance with Ethical Standards}

Funding: We acknowledge funding for part of this work from the Royal Society of Chemistry (RF188876).

Conflict of Interest: the author declares no conflict of interest.

Ethical approval: Bournemouth University ethics committee provided ethical approval for the work on ethyl glucuronide in fingernails.

Informed Consent: the author declares that informed consent was sought from all participants relating to this work. 


\section{References.}

[1] Pragst F, Balikova MA. State of the art in hair analysis for detection of drug and alcohol abuse. Clin Chim Acta. 2006; 370: 17-49.

[2] Pragst F. Interpretation problems in a forensic case of abstinence determination using alcohol markers in hair. Forensic Sci Int. 2012;10:217: 1-3.

[3] Society of Hair Testing: 2016 Consensus for the Use of Alcohol Markers in Hair for Assessment of both Abstinence and Chronic Excessive Alcohol Consumption.

http://www.soht.org/images/pdf/Revision\%202016_Alcoholmarkers.pdf. (Accessed 27.06.2018)

[4] Bossers LCAM, Paul R. Application of Bayesian theory to the reporting of results in alcohol hair testing. Forensic Sci Int. 2014; 242: e56-e58.

[5] Alladio E, Martyna A, Salomone A, Pirro V. Vincenti M, Zadora G. Evaluation of direct and indirect ethanol biomarkers using a likelihood ratio approach to identify chronic alcohol abusers for forensic purposes. Forensic Sci Int. 2017; 271:13-22.

[6] Biedermann A, Taroni F, Bozza S, Augsburger M, Aitken CGG. Critical analysis of forensic cut-offs and legal thresholds: A coherent approach to inference and decision. Forensic Sci Int. 2018; 288: 7280.

[7] Cappelle D, Neels H, De Keukeleire S, Fransen E, Dom G, Vermassen A, et al. Ethyl glucuronide in keratinous matrices as biomarker of alcohol use: A correlation study between hair and nails.

Forensic Sci Int. 2017; 279: 187-191. 